\title{
Line Width Mathematical Model in Fused Deposition Modelling for Precision Manufacturing
}

\author{
JC Jiang, Xinghua Xu*, Wanzhi Rui, Zhengrong Jia, Zuowei Ping \\ National Key Laboratory of Science and Technology on Vessel Integrated Power System, Wuhan, China
}

\begin{abstract}
Additive manufacturing is becoming increasingly popular because of its unique advantages, especially fused deposition modelling (FDM) which has been widely used due to its simplicity and comparatively low price. However, in current FDM processes, it is difficult to fabricate parts with highly accurate dimensions. One of the reasons is due to the slicing process of 3D models. Current slicing software divides the parts into layers and then lines (paths) based on a fixed value. However, in a real printing process, the printed line width will change when the process parameters are set in different values. The various printed widths may result in inaccuracy of printed dimensions of parts if using a fixed value for slicing. In this paper, a mathematical model is proposed to predict the printed line width in different layer heights. Based on this model, a method is proposed for calculating the optimal width value for slicing 3D parts. In the future, the proposed mathematical model can be integrated into slicing software to slice $3 \mathrm{D}$ models for precision additive manufacturing.
\end{abstract}

\section{Introduction}

3D printing technologies (also known as additive manufacturing (AM)) have been developed for more than twenty years. This technology has become a competitive technique for manufacturing parts with complex structures that are difficult or even impossible to be produced via conventional manufacturing technologies [1-4]. Fused deposition modelling (FDM) is one of the most common AM technologies for manufacturing 3D parts. It has been widely used for fabricating not only prototypes, but also real life products. The principle of FDM is based on the manner of layer-by-layer printing and each layer is printed in a line-by-line manner [5-11]. Figure 1 shows the process of slicing 3D models for fabrication. Once a 3D model is obtained, it needs to be sliced into layers and each layer will be sliced into lines as shown in Figure 1. Then the final sliced 3D model part can be obtained, which will be sent to the printers for fabrication.

Tamburrino et al. [12] studied the influence of slicing parameters on the multi-material adhesion mechanisms of FDM printed parts. Different slicing parameters will affect the final printed properties. We previously [13-17] also studied different parameters' effects on printable overhang and bridge features. We also [18-20] proposed some new printing methods via improving path planning process. Luu et al. [21] proposed an efficient slicing method for Catmull-Clark solids with functionally graded material. A new slicing algorithm was proposed by Wang and $\mathrm{Li}$ [22] to guarantee non-negative error of parts fabricated in AM processes. A novel toolpath generation method was proposed by Flores et al. [23], with the aim of fabricating metallic components in laser metal deposition technique. Volpato and Zanotto [24] studied the influence of different deposition sequences on final printed properties in FDM. Ezair et al. [25] proposed a volumetric covering print-path slicing for printing 3D models with superior properties (such as mechanical strength and surface finish). Currently, Xiong et al. [26] proposed a process planning method for adaptive contour parallel toolpath in AM with variable bead widths. The reasons of carrying out the above studies are mainly because of that the slicing parameters in AM will influence the final printed properties and accuracy. Slicing parameters change in each fabrication process may lead to unstable results of $3 \mathrm{D}$ printed properties and dimension accuracy. As shown in Figure 1(b), will the designed width of the part ( $\left.W_{\text {designed }}\right)$ equal the final fabricated width $(5 \times w)$ ? In fact, it is hard to achieve $W_{\text {designed }}=5 \times \mathrm{w}$ in real fabrication processes due to the change of process parameters.

In this paper, a mathematical model is proposed to predict the printed line width in different process parameters (layer height, print speed, filament extrusion speed) for making the fabrication more stable. Based on this model, a method is proposed for calculating the optimal width value for slicing $3 \mathrm{D}$ parts. The mechanism of printed line width in AM processes are studied. In the future, the proposed mathematical model can be integrated into slicing software to slice $3 \mathrm{D}$ models for precision additive manufacturing.

* Corresponding author: xinghuaxv@163.com 


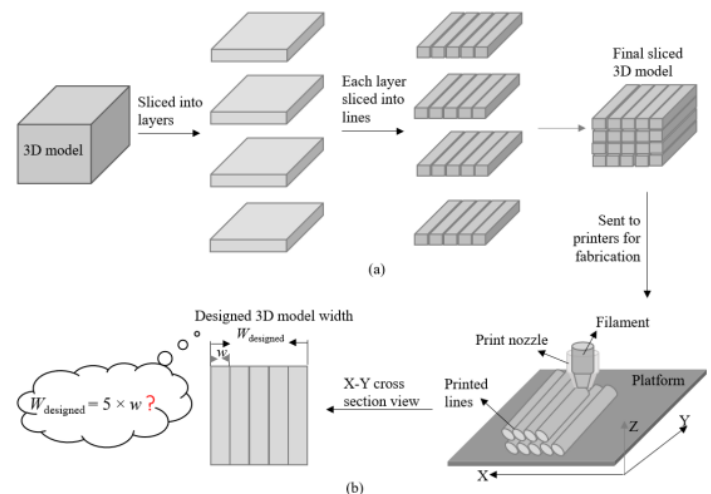

Figure 1. (a) Process of slicing 3D models into layers and lines for printing; (b) fabrication process and illustration of slicing width

\section{Mathematical model}

\subsection{Model for predicting printed line width in different process parameters}

In $\mathrm{AM}$ fabrication processes, the printed line widths will change when process parameters change, especially layer height, print speed and filament extrusion speed. In a certain situation (all the process parameters keep the same while changing the layer height), the final printed line width will change as shown in Figure 2. This is due to the volume of extruded material keeps the same in certain process parameters and when changing the layer height, the widths will change. Larger layer height may lead to shorter width of printed layer due to extruded material volume consistency in certain filament extrusion speed. In time ' $t$ ', the volume $\left(M_{\text {extruded }}\right)$ of extruded material can be calculated as follows:

$M_{\text {extruded }}=\pi\left(\frac{D_{\text {filament }}}{2}\right)^{2} v_{\text {filament }} t$

where $\pi$ is approximately equal to $3.14159 ; D_{\text {filament }}$ is the diameter of filament material; $v_{\text {filament }}$ is the speed of filament extrusion speed. The length of the printed line during the period $t$ can be calculate as the print speed $v_{\text {print }}$ times $t$ as shown in Figure 3. Therefore, the cross section area $S_{\mathrm{T}}$ in Figure 3 can be calculated as:

$S_{T}=\frac{\pi\left(\frac{D_{\text {filament }}}{2}\right)^{2} v_{\text {filament }} t}{v_{\text {print }} t}=\frac{\pi\left(\frac{D_{\text {filament }}}{2}\right)^{2} v_{\text {filament }}}{v_{\text {print }}}$

As can be seen in this equation, the cross section area $S_{\mathrm{T}}$ will change as filament extrusion speed and/or print speed change.

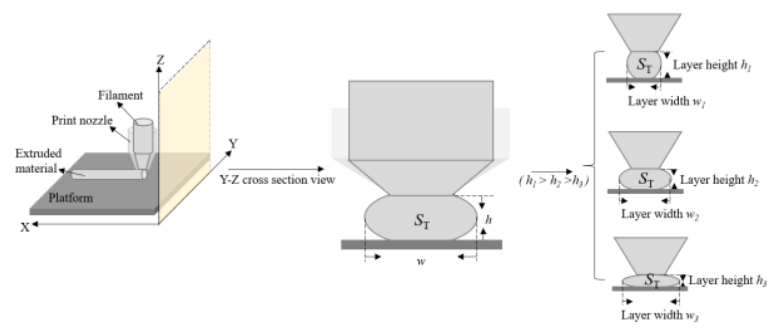

Figure 2. Illustration of line width change in different layer heights

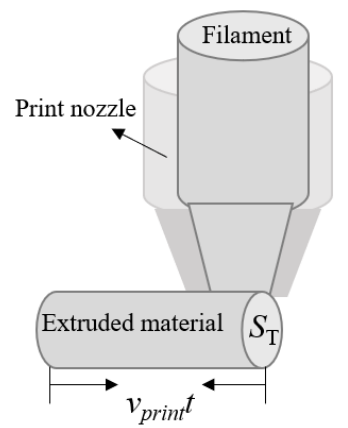

Figure 3. Printed length of line during a period $t$ and the cross section area $\mathrm{S}_{\mathrm{T}}$

Put the cross section geometry of extruded material in Figure 4(a) into a Cartesian coordinate system as shown in Figure 4(b). Then the oval geometry curve can be represented as:

$\frac{x^{2}}{a^{2}}+\frac{y^{2}}{h^{2}}=1$

where $a$ and $b$ are the half lengths of the two axes as shown in Figure 4(b). The point $\left(\frac{D_{\text {nozzle }}}{2}, \frac{h}{2}\right)$ should be on the oval curve as shown in Figure 4(b). That means it is true when substituting

$\left\{\begin{array}{c}\mathrm{x}=\frac{h}{2} \\ \mathrm{y}=\frac{D_{\text {nozzle }}}{2}\end{array}\right.$

into Equation (3). Then the following equation can be derived.

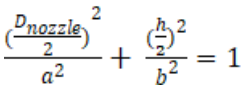

As shown in Equation 2, the value of cross section area keeps a constant regardless of the layer height. When the layer height is $h$ as shown in Figure 4(b), the cross section area $S_{\mathrm{T}}$ can be calculated as

$S_{T}=\pi a b-2 *\left(2 \int_{-b}^{\frac{2 b-h}{2}-b} \frac{a}{b} \sqrt{b^{2}-y^{2}} d y\right)=$

$\pi a b-2 *\left(\frac{a}{b}[(h-b) \sqrt{h(2 b-h)}+\right.$

$\left.b^{2} \arcsin \frac{h-b}{b}+\frac{1}{2} \pi b^{2}\right]$ )

Combining equations (2), (5) and (6), the values of $a$ and $b$ can be obtained. Then the printed line width in different layer heights can be obtained as

$w=2 a$ 


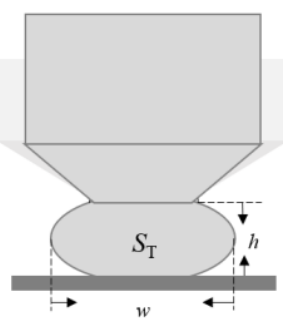

(a)

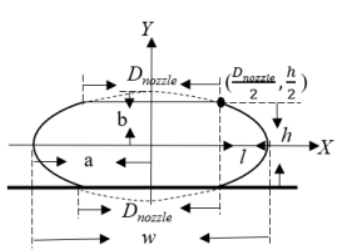

(b)
Figure 4. Geometry and dimension illustration for calculating $w$

\subsection{Model for calculating optimal width for slicing parts}

During the process of slicing 3D models, the width used for dividing a layer into lines is set as a constant by the slicing software. Here, the value of width is different from the printed line width. The width $\left(w_{c}\right)$ here is the value used for slicing layers. This value of width should be changed when the layer height changes as it is similar as discussed in the previous section. In this subsection, a mathematical model is proposed for calculating the optimal width that should be used for slicing layers. As shown in Figure 5, the value of overlapped area changes when using different widths to slice a layer. The smaller the width is, the more the overlapped area. Once the overlapped area is larger than a value, the material may overflow as shown in Figure 5(c) and (d). While if the overlapped area is smaller than a value, space gap may occur between two printed lines as shown in Figure 5(a) and (b). The optimal overlapped area and corresponding material distribution between two printed lines should be as shown in Figure 6, ideally no space gap and overflow. Theoretically speaking, this can be achieved when the following equation is true.

$S_{E}=S_{E 1}+S_{E 2}$

where $S_{E}$ is the size of overlapped area $E$ in Figure 6, $S_{E 1}$ and $\mathrm{S}_{\mathrm{E} 2}$ are the sizes of areas E1 and E2 in Figure 6, respectively. In Figure 6, $\mathrm{S}_{\mathrm{E}}, \mathrm{S}_{\mathrm{E} 1}$ and $\mathrm{S}_{\mathrm{E} 2}$ can be calculated as follows.

$$
\begin{aligned}
& S_{E}=2 *\left(2 \int_{-a}^{\frac{-w_{c}}{2}} \frac{b}{a} \sqrt{a^{2}-x^{2}} d x\right) \\
& =2 * \frac{b}{a}\left[-\frac{w_{c}}{2} \sqrt{\left(a-\frac{w_{c}}{2}\right)\left(a+\frac{w_{c}}{2}\right)}+\right. \\
& a^{2} \arcsin \left(-\frac{w_{c}}{2 a}\right)+\frac{1}{2} \pi a^{2}
\end{aligned}
$$

$S_{E 1}=S_{E 2}=\frac{h w_{c}-2 * \frac{h D_{\text {nozzle }}}{2}-\left(2 * 2 \int_{-a}^{-\frac{-D_{\text {nazzle }}}{2}} \frac{b}{a} \sqrt{a^{2}-x^{2}} d x-S_{E}\right)}{2}$

$=\frac{1}{2} h w_{c}-\frac{h D_{\text {nozzle }}}{2}-$

$\frac{b}{a}\left[\frac{-D_{\text {nozzle }}}{2} \sqrt{\left(a-\frac{D_{\text {nozzle }}}{2}\right)\left(a+\frac{D_{\text {nozzle }}}{2}\right)}+\right.$

$a^{2} \arcsin \left(\frac{-D_{\text {nozzle }}}{2 a}\right)+\frac{1}{2} \pi a^{2} \mid+\frac{1}{2} S_{E}$
Based on Equations (8), (9) and (10), the optimal width $w_{c}$ for slicing can be obtained.

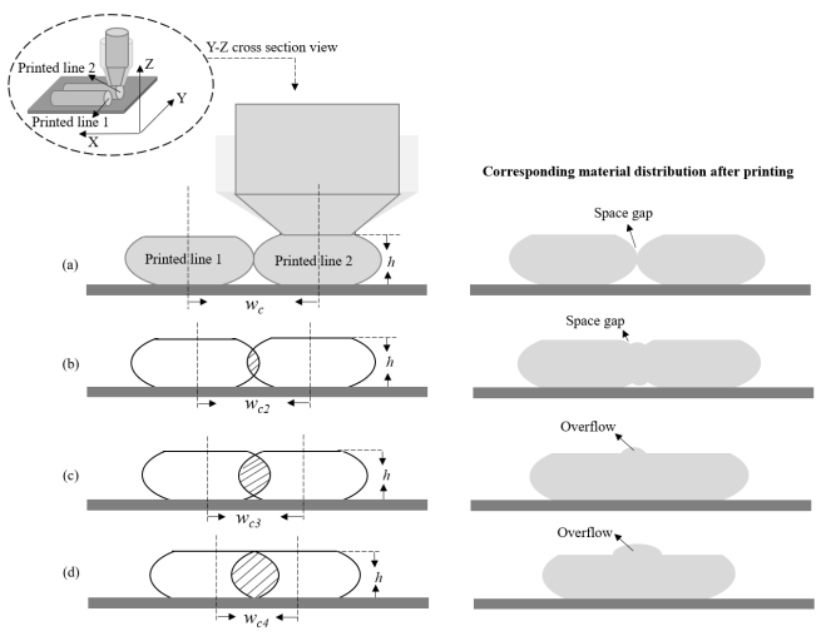

Figure 5. Overlapped area (dashed) and material distribution illustration when using different widths for slicing layers

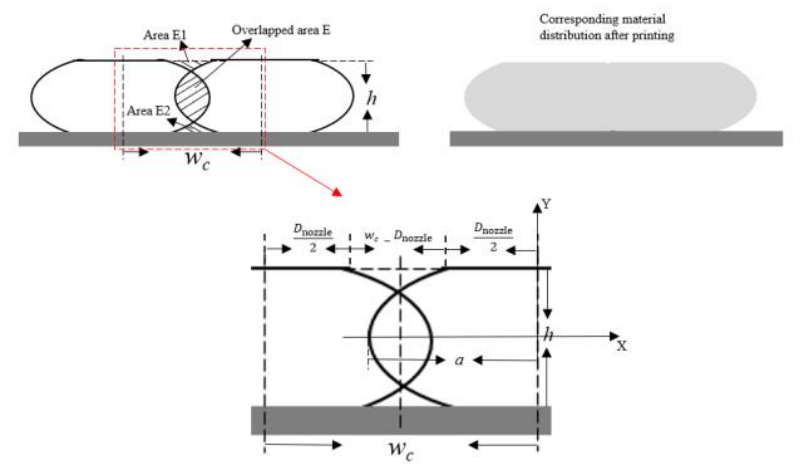

Figure 6. Optimal overlapped area and corresponding material distribution between two printed lines

\section{Conclusion}

In this paper, a mathematical model is proposed to predict the printed line width in different layer heights. Based on this model, a method is proposed for calculating the optimal width value for slicing $3 \mathrm{D}$ parts. In the future, the proposed mathematical model can be integrated into slicing software to slice 3D models for precision additive manufacturing. Currently, however, this is only a theoretical investigation. Experiments and comparisons will be carried out in the future.

\section{References}

1. Liu J, Gaynor AT, Chen S, et al (2018) Current and future trends in topology optimization for additive manufacturing. Struct Multidiscip Optim 57:24572483 . doi: 10.1007/s00158-018-1994-3

2. Jiang J, Stringer J, Xu X, Zheng $\mathrm{P}$ (2018) A benchmarking part for evaluating and comparing support structures of additive manufacturing. In: 3rd International Conference on Progress in Additive Manufacturing (Pro-AM 2018). pp 196-202 
3. Fu Y-F, Rolfe B, Chiu LNS, et al (2019) Design and experimental validation of self-supporting topologies for additive manufacturing. Virtual Phys Prototyp 14:382-394 . doi: 10.1080/17452759.2019.1637023

4. Jiang J, Xu X, Jonathan Stringer (2019) Effect of Extrusion Temperature on Printable Threshold Overhang in Additive Manufacturing. In: CIRP Manufacturing Systems Conference 2019. Ljubljana

5. Jiang J, Stringer J, Xu X (2019) Support Optimization for Flat Features via Path Planning in Additive Manufacturing. 3D Print Addit Manuf 6:171-179 . doi: 10.1089/3dp.2017.0124

6. Jiang J, Xu X, Stringer J (2018) Support Structures for Additive Manufacturing: A Review. J Manuf Mater Process 2:64 . doi: 10.3390/jmmp2040064

7. Jiang J (2020) A novel fabrication strategy for additive manufacturing processes. J Clean Prod 272:122916 . doi: 10.1016/j.jclepro.2020.122916

8. Jiang J, Xu X, Xiong Y, et al (2020) A novel strategy for multi-part production in additive manufacturing. Int J Adv Manuf Technol 109:1237-1248 . doi: 10.1007/s00170-020-05734-8

9. Jiang J, Ma Y (2020) Path planning strategies to optimize accuracy, quality, build time and material use in additive manufacturing: A review. Micromachines 11:633

10. Jiang J, Yu C, Xu X, et al (2020) Achieving better connections between deposited lines in additive manufacturing via machine learning. Math Biosci Eng 17:3382-3394

11. Yu C, Jiang J (2020) A Perspective on Using Machine Learning in 3D Bioprinting. Int $\mathbf{J}$ Bioprinting 6:4-11 . doi: 10.18063/ijb.v6i1.253

12. Tamburrino F, Graziosi S, Bordegoni M (2019) The influence of slicing parameters on the multi-material adhesion mechanisms of FDM printed parts: an exploratory study. Virtual Phys Prototyp. doi: 10.1080/17452759.2019.1607758

13. Jiang J, Stringer J, Xu X, Zhong RY (2018) Investigation of printable threshold overhang angle in extrusion-based additive manufacturing for reducing support waste. Int J Comput Integr Manuf 31:961-969 . doi: 10.1080/0951192X.2018.1466398

14. Jiang J, Lou J, Hu G (2019) Effect of Support on Printed Properties in Fused Deposition Modelling Processes. Virtual Phys Prototyp 14:308-315 . doi: 10.1080/17452759.2019.1568835

15. Jiang J, Weng F, Gao S, et al (2019) A Support Interface Method for Easy Part Removal in Direct Metal Deposition. Manuf Lett 20:30-33 . doi: 10.1016/j.mfglet.2019.04.002
16. Jiang J, Hu G, Li X, et al (2019) Analysis and prediction of printable bridge length in fused deposition modelling based on back propagation neural network. Virtual Phys Prototyp 14:253-266 . doi: 10.1080/17452759.2019.1576010

17. Weng F, Gao S, Jiang J, et al (2019) A novel strategy to fabricate thin $316 \mathrm{~L}$ stainless steel rods by continuous direct metal deposition in $\mathrm{Z}$ direction. Addit Manuf 27:474-481 . doi: 10.1016/j.addma.2019.03.024

18. Jiang J, Xu X, Stringer J (2019) Optimisation of multi-part production in additive manufacturing for reducing support waste. Virtual Phys Prototyp 14:219-228 . doi: 10.1080/17452759.2019.1585555

19. Jiang J, Xu X, Stringer J (2019) Optimization of Process Planning for Reducing Material Waste in Extrusion Based Additive Manufacturing. Robot Comput Integr Manuf 59:317-325 . doi: 10.1016/j.rcim.2019.05.007

20. Jiang J, Xu X, Stringer J (2018) A new support strategy for reducing waste in additive manufacturing. In: The 48th International Conference on Computers and Industrial Engineering (CIE 48). Auckland, pp $1-7$

21. Luu TH, Altenhofen C, Ewald T, et al (2019) Efficient slicing of Catmull-Clark solids for 3D printed objects with functionally graded material. Comput Graph. doi: 10.1016/j.cag.2019.05.023

22. Wang $\mathrm{Y}, \mathrm{Li} \mathrm{W}$ (2019) A slicing algorithm to guarantee non-negative error of additive manufactured parts. Int J Adv Manuf Technol. doi: 10.1007/s00170-018-3199-8

23. Flores J, Garmendia I, Pujana J (2019) Toolpath generation for the manufacture of metallic components by means of the laser metal deposition technique. Int J Adv Manuf Technol. doi: 10.1007/s00170-018-3124-1

24. Volpato N, Zanotto TT (2019) Analysis of deposition sequence in tool-path optimization for low-cost material extrusion additive manufacturing. Int $\mathrm{J}$ Adv Manuf Technol. doi: 10.1007/s00170-018-3108-1

25. Ezair B, Fuhrmann S, Elber G (2018) Volumetric covering print-paths for additive manufacturing of 3D models. CAD Comput Aided Des 100:1-13 . doi: 10.1016/j.cad.2018.02.006

26. Xiong Y, Park SI, Padmanathan S, et al (2019) Process planning for adaptive contour parallel toolpath in additive manufacturing with variable bead width. Int J Adv Manuf Technol. doi: 10.1007/s00170-019-03954-1 